\title{
A Rare Case of Systemic Mastocytosis With Associated Clonal Hematological Non-Mast Cell Lineage Disease That Transformed to Acute Leukemia With IDH2 Mutation
}

\author{
Andrew D. Liman ${ }^{a}$, b, e, Jenna Shields ${ }^{c}$, Agnes K. Liman ${ }^{\mathrm{d}}$
}

\begin{abstract}
An elderly 72-year-old man presented with anemia, thrombocytopenia, monocytosis, splenomegaly and lymphadenopathy. Bone marrow biopsy was consistent with mast cell neoplasm with positive CD117, $\mathrm{CD} 25, \mathrm{CD} 34$ myeloblasts and polymerase chain reaction (PCR) revealed mutation of D816V. He developed bilateral femoral neck fractures and biopsy confirmed that he has systemic mastocytosis (SM). He received cladribine and midostaurin with stable disease for 21 months. His SM with associated clonal hematological non-mast cell lineage disease (SM-AHNMD) transformed to acute myelogenous leukemia with isocitrate dehydrogenase 2 (IDH2) mutation. A trial of enasidenib was given for 5 months but without any response. Patient decided to go with home hospice and died afterwards.
\end{abstract}

Keywords: SM-AHNMD; KIT D816V mutation; Serum tryptase; Acute myelogenous leukemia; Midostaurin; IDH2 mutation; Enasidenib

\section{Introduction}

Systemic mastocytosis (SM) is a rare myeloid neoplasm that is caused by accumulation of abnormal mast cells in the bone marrow (BM), liver, spleen, and skin. The KIT D816V mutation encodes a constitutively activated receptor tyrosine kinase that drives disease pathogenesis. The KIT D $816 \mathrm{~V}$ mutation is the most common genetic alteration of SM which occurred in

Manuscript submitted July 27, 2020, accepted August 4, 2020

Published online August 28, 2020

aHematology and Oncology, VA Central California Health Care System, Fresno, CA, USA

bUniversity of California San Francisco at Fresno, Fresno, CA, USA

cPharmacy, VA Pittsburgh Healthcare System, Pittsburgh, PA, USA

dPathology and Laboratory Medicine, VA Central California Health Care System, Fresno, CA, USA

${ }^{\text {e}}$ Corresponding Author: Andrew D. Liman, Hematology and Oncology, VA Central California Health Care System, 2615 E. Clinton Ave, Fresno, CA 93703, USA. Email: Andrew.liman@va.gov

doi: https://doi.org/10.14740/jmc3552 about $90 \%$ of SM with associated clonal hematological nonmast cell lineage disease (SM-AHNMD). Advanced SM is associated with a poor prognosis $[1,2]$. We present a rare case of SM-AHNMD that transformed to acute myelogenous leukemia (AML) with isocitrate dehydrogenase 2 (IDH2) mutation.

\section{Case Report}

A 72-year-old male patient presented in August 2016 with anemia (hemoglobin (HGB) $12.2 \mathrm{~g} / \mathrm{dL}$ ), thrombocytopenia (platelet $86,000 / \mu \mathrm{L}$ ), absolute monocyte count of 2,000 $4,000 / \mu \mathrm{L}$ and weight loss. Computed tomography (CT) scan showed splenomegaly and lymphadenopathy. BM biopsy was positive for CD117 mast cells and CD34 myeloblasts. Immunohistochemistry (IHC) confirmed that the neoplastic cells were positive for CD25 (Fig. 1a-e). Polymerase chain reaction (PCR) analysis revealed KIT D816V point mutation which established the diagnosis of SM. Serum tryptase at diagnosis was $295 \mathrm{ng} / \mathrm{mL}$ (normal $2.2-13.2 \mathrm{ng} / \mathrm{mL}$ ). He initially refused chemotherapy, and thus was started on imatinib mesylate. In April 2017, he developed bilateral femoral neck fractures. Hip biopsy was consistent with SM (positive CD25 and CD117) with dysplastic megakaryocytes and increased monocytosis. $\mathrm{He}$ received four cycles of cladribine with good response in splenomegaly, anemia and thrombocytopenia. Serum tryptase initially decreased to $141 \mathrm{ng} / \mathrm{mL}$ but then rose up to $243 \mathrm{ng} /$ $\mathrm{mL}$. Midostaurin $100 \mathrm{mg}$ orally twice a day was initiated in December 2017. His cytopenia and splenomegaly were improved. By June 2018 his platelet increased to $135,000 / \mu \mathrm{L}$. He was continued on midostaurin with stable disease until September 2019 when his pancytopenia significantly worsened. His white blood cells (WBCs) decreased to $2,000 / \mu \mathrm{L}$, HGB to $6.9 \mathrm{~g} / \mathrm{dL}$ and platelet to $28,000 / \mu \mathrm{L}$ (Table 1). BM biopsy on October 2019 revealed increased blasts of $24 \%$ consistent with AML. FLT3 and NPM1 mutation were negative. IHC for CD25 was positive in scattered mast cells. Next-generation sequencing (NGS) analysis revealed positive for $\mathrm{IDH} 2$ mutation. Enasidenib $100 \mathrm{mg}$ daily was initiated in November 2019. After 5 months of enasidenib there was no improvement in weekly needs for blood and platelet transfusion (Fig. 2). Peripheral blood smear in March 2020 showed more than 50\% blasts; thus, enasidenib was discontinued. The patient decided to go with home hospice and died in April 2020. 


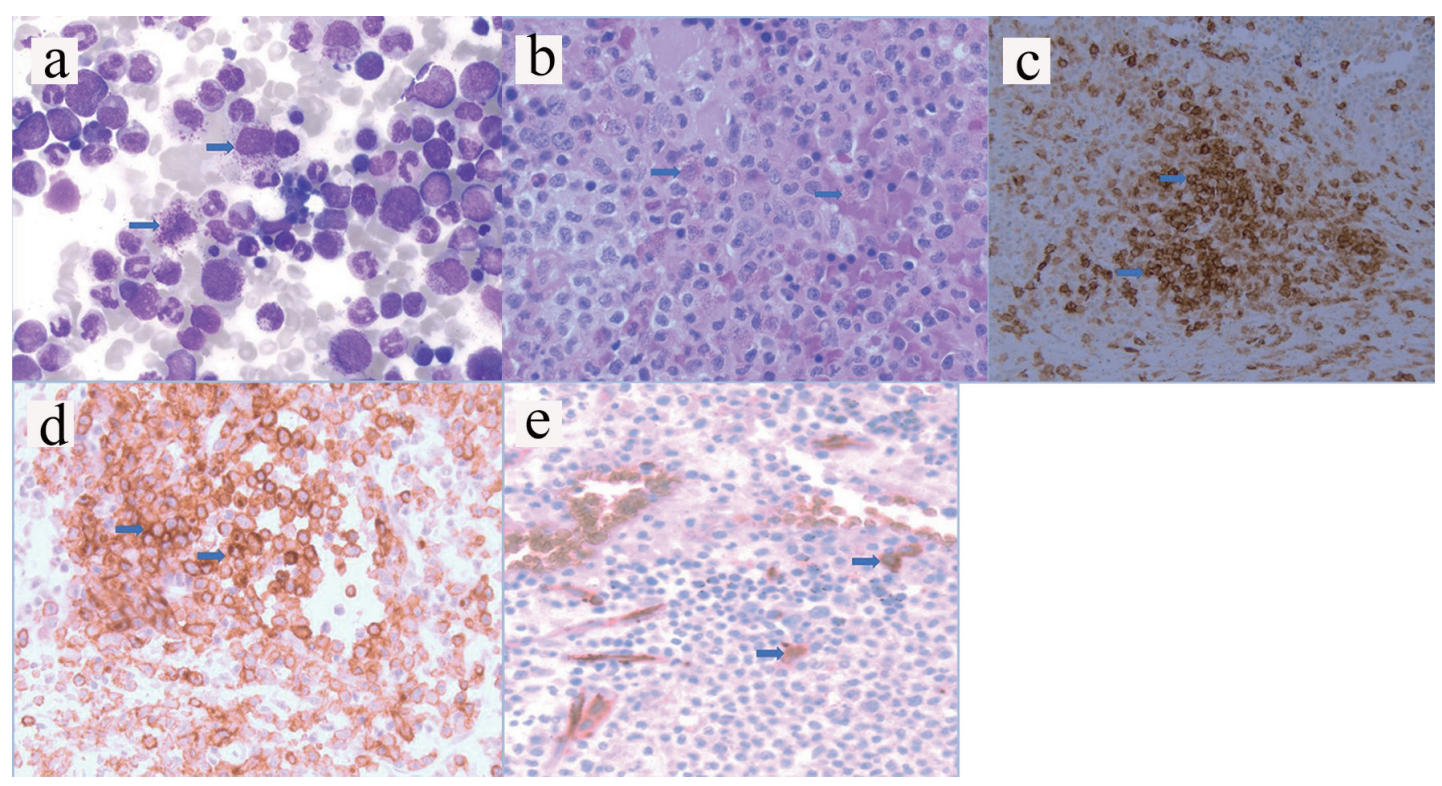

Figure 1. (a) Hematoxylin and eosin staining from aspirate (arrows pointed to mast cells). (b) Hematoxylin and eosin staining from biopsy (arrows pointed to mast cells). (c) Immunostaining positive for CD117 (arrows pointed to brown colored cells). (d) Immunostaining positive for CD25 (arrows pointed to brown colored cells). (e) Immunostaining positive for CD34 myeloblasts (arrows pointed to brown colored cells).

Table 1. Response of CBC and Serum Tryptase Over Time

\begin{tabular}{|c|c|c|c|c|c|c|c|}
\hline Lab result & $8 / 30 / 16$ & $11 / 7 / 16$ & $9 / 20 / 17$ & $11 / 13 / 17$ & $6 / 14 / 18$ & 9/1/19 & $3 / 16 / 20$ \\
\hline WBC $($ per $\mu \mathrm{L})(4,800-11,000)$ & 11,100 & 12,700 & 8,100 & 21,200 & 9,800 & 2,000 & 8,000 \\
\hline $\operatorname{HGB}(\mathrm{g} / \mathrm{dL})(14.0-18.0)$ & 12.2 & 11.2 & 13.7 & 14.7 & 10.7 & 6.9 & 7.7 \\
\hline Tryptase $(\mathrm{ng} / \mathrm{mL})(2.2-13.2)$ & - & 295 & 141 & 243 & 275 & 124 & 5.3 \\
\hline
\end{tabular}

WBC: white blood cell; HGB: hemoglobin; CBC: complete blood count.

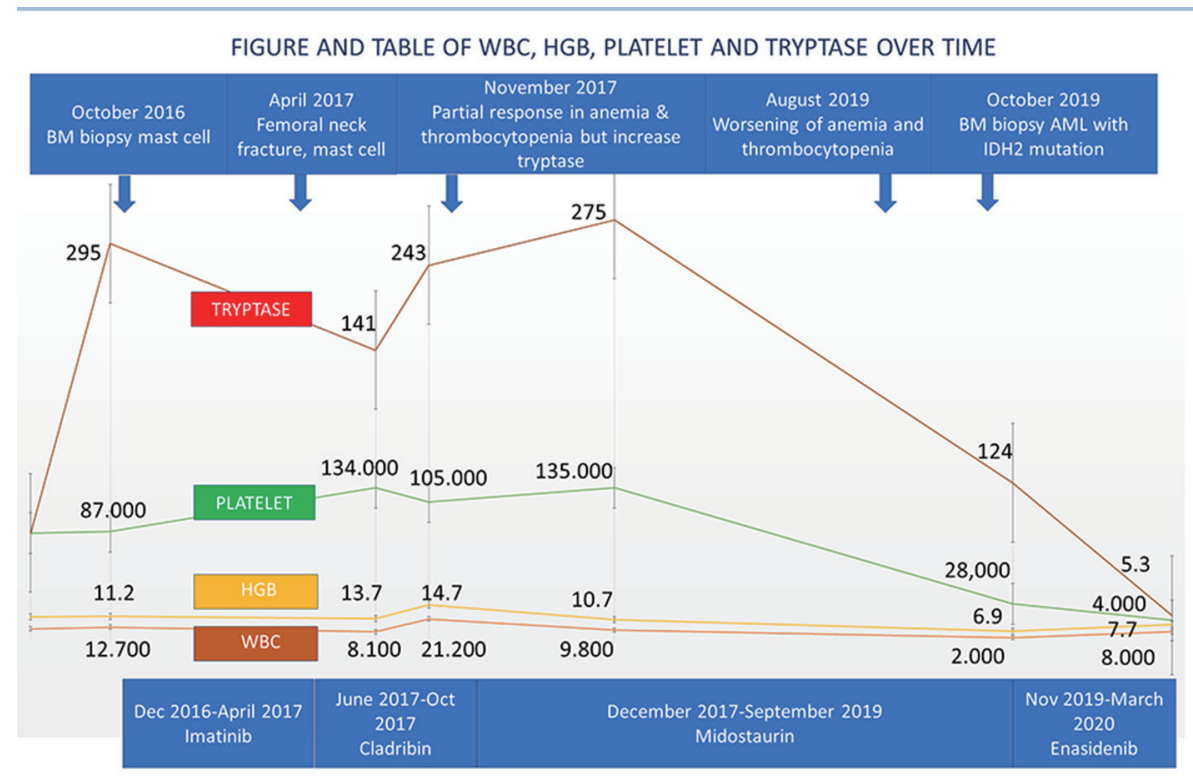

Figure 2. Disease history of WBC, HGB, platelet and tryptase over time. WBC: white blood cell; HGB: hemoglobin. 


\section{Discussion}

The diagnosis of SM is established using SM criteria provided by the World Health Organization (WHO). At least one major and one minor or at least three minor SM criteria must be fulfilled to establish the diagnosis of SM. Major SM criteria consist of multifocal dense infiltrates of mast cells ( $>15$ mast cells in aggregates) in BM biopsy or extracutaneous organ. Minor SM consists of either: 1) $>25 \%$ of all mast cells are atypical cells (type I or type II) on BM smears or are spindleshaped in mast cell infiltrates; 2) KIT point mutation at codon 816 in the BM or another extracutaneous organ; 3) Mast cells in $\mathrm{BM}$ or blood or another extracutaneous organ exhibit CD2 and/or CD25; or 4) Baseline serum tryptase level > $20 \mathrm{ng} /$ $\mathrm{mL}$. The C-findings include BM involvement and cytopenias, liver-function abnormalities, hypoalbuminemia, weight loss, ascites, and osteolytic bone lesions [2, 3]. Our patient fulfilled the criteria of SM-AHNMD based on major and minor criteria, C-findings, monocytosis and dysplastic megakaryocytes. He most likely has chronic myelomonocytic leukemia which is an associated clonal hematological non-mast cell disease. He did not respond to imatinib because of KIT D816V mutation. He achieved a partial response with cladribine.

Midostaurin is an oral multi-kinase inhibitor with activity against multiple protein including FLT3 and KIT D816V mutation. In a phase II study of SM including AHNMD, the overall response rate was $60 \%[1,3]$. Our patient responded well with midostaurin without any significant toxicity for 21 months, but his SM transformed to AML with $I D H 2$ mutation. Jawhar et al were able to prove in a multivariate analysis that only KIT D816V reduction $>25 \%$ was an independent marker for improved overall survival. Secondary mechanism leading to progression was associated with emergence mutations in RUNX1, K/NRAS or IDH2 [4].

IDH 2 gene mutation was reportedly occurred in $12 \%$ of AML and 5\% of myelodysplastic patients. IDH2 mutation causes aberrant DNA hypermethylation and blocks differentiation of myeloid precursors. This mutation will ultimately promote leukemogenesis. Enasidenib is an oral small molecule selective targeted inhibitor of the mutant IDH2 enzyme. A phase I/II trial patients with relapsed AML received enasidenib $100 \mathrm{mg}$ daily with overall response rate of $40 \%$ and a median response duration of 5.8 months [5]. Our patient unfortunately did not respond to this drug after 5 months of therapy. Other treatment options for SM-AHNMD that is related with myelodysplasia include hypomethylating agents such as azacytidine or decitabine.

\section{Conclusions}

SM-AHNMD constitutes approximately $40 \%$ of all SM with poor prognosis. SM is resistant to imatinib because of KIT D816V mutation. Cladribine has some activity. Midostaurin has good response that can stabilize the disease for several months. NGS analysis is very important in determining secondary mutation including $I D H 2$ mutation. Most patients with transformation to AML have poor prognosis. Hematologists should be able to diagnose SM and treat the disease based on mutational analysis.

\section{Acknowledgments}

None to declare.

\section{Financial Disclosure}

None to declare.

\section{Conflict of Interest}

None to declare.

\section{Informed Consent}

The manuscript has been de-identified. Patient signed informed consent.

\section{Author Contributions}

All authors contributed to the editing of the manuscript. ADL wrote the manuscript, AKL provided pathology images and JS provided information about medications. All authors reviewed the manuscript.

\section{Data Availability}

The authors declare that the data supporting the findings of this study are available within the article. We will provide more data upon request.

\section{References}

1. Gotlib J, Kluin-Nelemans HC, George TI, Akin C, Sotlar K, Hermine O, Awan FT, et al. Efficacy and safety of midostaurin in advanced systemic mastocytosis. N Engl J Med. 2016;374(26):2530-2541.

2. Valent P, Akin C, Metcalfe DD. Mastocytosis: 2016 updated WHO classification and novel emerging treatment concepts. Blood. 2017;129(11):1420-1427.

3. Valent P, Sperr WR, Akin C. How I treat patients with advanced systemic mastocytosis. Blood. 2010;116(26):58125817.

4. Jawhar M, Schwaab J, Naumann N, Horny HP, Sotlar K, Haferlach T, Metzgeroth G, et al. Response and progression on midostaurin in advanced systemic mastocytosis: KIT D816V and other molecular markers. Blood. 2017;130(2):137-145.

5. Stein EM, DiNardo CD, Pollyea DA, Fathi AT, Roboz GJ, Altman JK, Stone RM, et al. Enasidenib in mutant IDH2 relapsed or refractory acute myeloid leukemia. Blood. 2017;130(6):722-731. 